\title{
ANATOMÍA QUIRÚRGICA DE NERVIOS FACIAL Y AURICULAR MAYOR DEL CONEJO COMO MODELO EXPERIMENTAL
}

\author{
Juan Carlos Izquierdo Velásquez MD*, Ángela María Campos Mahecha MD**
}

\section{Resumen}

\section{Resumen}

Objetivo: describir la anatomía quirúrgica de los nervios facial y auricular mayor del conejo como modelo experimental. Técnica quirúrgica: disección bilateral de los dos nervios en doce conejos. Se describen el patrón de distribución, reparos quirúrgicos y similitudes y diferencias con humanos. Resultados: el facial viaja por debajo del sistema musculoaponeurótico superficial y de la parótida, para dividirse en tres ramas principales: orbitaria, bucal y marginal. El auricular mayor es posterior al facial y la vena retrofacial es el reparo principal. Ambos son nervios polifasciculares. Conclusiones: el facial del conejo es un modelo para estudios de regeneración nerviosa dada su configuración polifascicular semejante al humano. El nervio auricular mayor, para injerto, es accesible en el mismo campo quirúrgico.

Palabras clave: modelos animales experimentales, nervio facial, regeneración nerviosa.

\section{SURGICAL ANATOMY OF THE FACIAL AND GREAT AURICULAR NERVES IN A RABBIT EXPERIMENTAL MODEL}

\begin{abstract}
Objective: to describe the surgical anatomy of the facial and great auricular nerves in a rabbit experimental model. Surgical technique: bilateral dissection of both studied nerves in 12 rabbits. The anatomic distribution pattern and surgical landmarks as well as similarities and differences with human beings are described. Results: the facial nerve courses under the superficial muscular aponeurotic system and the parotid, and divides into three main branches: orbital, buccal and marginal branches. The great auricular nerve runs posterior to the facial nerve and the retrofacial vein constitutes the main landmark. Both have a multifasicular pattern. Conclusions: the facial nerve of the rabbit is a study model for nerve regeneration due to its multifascicular configuration similar to that in humans. The major auricular nerve is a study model for grafts and is accessible in the same surgical field.
\end{abstract}

Key words: experimental animal models, facial nerve, nerve regeneration.

Fecha recibido: diciembre 13 de 201।. Fecha aceptado: mayo 25 de 2012

* Profesor Asociado, Fundación Universitaria de Ciencias de la Salud. Servicio de Otología y Otoneurología, Hospital de San José. Profesor Asociado del Departamento de Cirugia, Unidad de Otorrinolaringología, Facultad de Medicina, Universidad Nacional de Colombia. Bogotá DC, Colombia.
** Otorrinolaringóloga de la Universidad Nacional de Colombia, Residente de segunda especialidad, Otologia y Otoneurologia, Fundación Universitaria Ciencias de la Salud. Bogotá DC, Colombia. 


\section{Introducción}

Mucho de nuestro entendimiento de la regeneración nerviosa se deriva de experimentos en modelos con animales pequeños, entre los más usados se incluyen el gato, el curí y la rata, cada uno con ventajas y desventajas. Los grandes son costosos y requieren más anestesia, pero en los animales tan pequeños la disección del nervio facial es más dispendiosa y las publicaciones respecto a su distribución son contradictorias.' En la literatura es posible encontrar estudios de nervio periférico en relación con suturas, injertos y efectos de la radiación usando nervios ciáticos de rata y conejo, entre otros, pero los estudios en el facial son menos frecuentes. ${ }^{1.2}$ El propósito de este estudio es describir el nervio facial del conejo como un modelo experimental y proponer al auricular mayor como injerto para estudios de regeneración nerviosa en lesiones severas del facial.

\section{Técnica quirúrgica}

Bajo anestesia general con xylasina en dosis de 10 $\mathrm{mg} / \mathrm{k}$ intramuscular y ketamina $50 \mathrm{mg} / \mathrm{k}$ por la misma vía en condiciones de asepsia y antisepsia y bajo magnificación, se realizó disección bilateral de los nervios facial y auricular mayor, en doce conejos hembra de raza Nueva Zelanda con un peso aproximado de 2.5 a $3 \mathrm{k}$. Todos fueron mantenidos en bioterio en condición estándar de vivienda, ventilación, alimentación, hidratación, temperatura, humedad y ciclo día/noche, teniendo en cuenta las disposiciones de la Ley 84 de 1989 por la cual se adopta el Estatuto Nacional de Protección de los Animales y el Título V de la Resolución 008430 de 1993, donde se establecen las normas de la investigación biomédica en animales. Fue aprobado por el comité de investigaciones de la Facultad de Medicina de la Fundación Universitaria de Ciencias de la Salud (FUCS) y el comité de ética de la Facultad de Medicina de la Universidad Nacional de Colombia. Para la disección se hizo una incisión desde la base auricular hasta el ángulo de la boca. Todos los conejos se estudiaron para la descripción de la anatomía. Se hizo registro fotográfico de las disecciones, lo cual permitió asegurar que las relaciones anatómicas y las proporciones descritas fueran precisas.

\section{Resultados}

\section{Anatomía macroscópica del nervio facial}

Tronco principal: bajo una delgada capa de piel y tejido celular subcutáneo se halló el sistema músculo aponeurótico superficial (SMAS) que a nivel del cuello se continúa con el músculo platisma. Todas las ramas periféricas se encontraron cubiertas por la fascia superficial y aplicadas sobre la cara lateral del músculo masetero (Figura 1).

El nervio facial se ubicó emergiendo del agujero estilomastoideo en parte lateral de la base de cráneo, $1.5 \mathrm{~cm}$ anteroinferior al conducto auditivo externo, por delante de la porción mastoidea del músculo cleidomastoideo.

El tronco principal tuvo un trayecto de 4 a $5 \mathrm{~mm}$ con dirección anterior. Tan pronto sale por el foramen estilomastoideo emerge la primera rama, el auricular posterior que se dirige hacia la parte superior y posterior para inervar los músculos de la oreja. Por tanto,

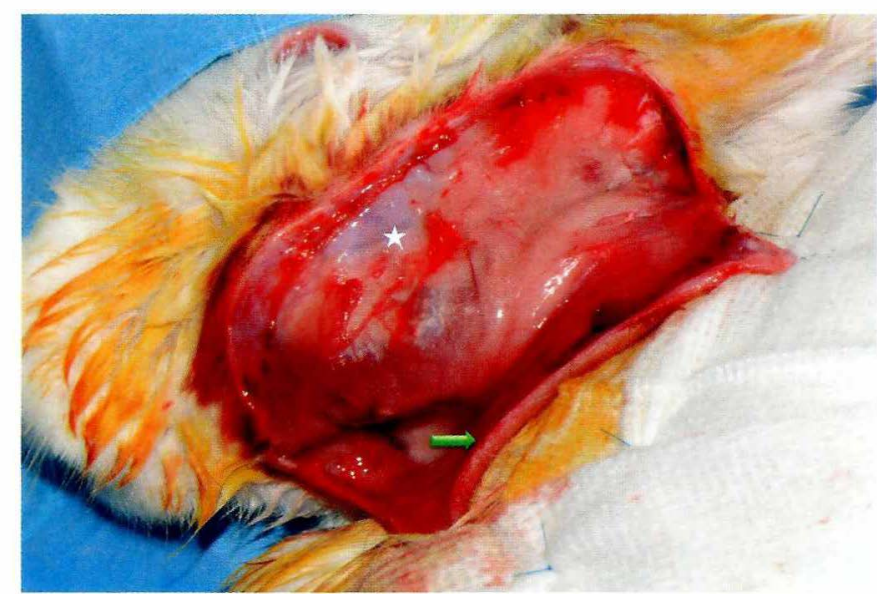

Figura I. Incisión de piel, tejido celular subcutáneo y SMAS (flecha verde señalando la piel y el tejido celular subcutáneo rechazado hacia la parte inferior). Se ha preservado la fascia superficial con las ramas del nervio facial protegidas por ella (estrella blanca). 
y para efectos prácticos, el tronco principal se define como la porción proximal a la emergencia de la rama auricular posterior hasta la bifurcación.

La vena facial posterior se halló con un calibre de 2.5 a $3 \mathrm{~mm}$, pasando medial al tronco principal del nervio facial y recibiendo por debajo de éste a la vena auricular posterior, para continuar hacia la porción inferior en la sustancia de la glándula parótida y unirse a nivel del ángulo de la mandíbula con la vena facial anterior para formar la vena yugular externa (Figura 2). Cuando fue necesario se disecó, ligó, coaguló o movilizó la vena facial posterior y sus tributarias (Figura 3).

Bifurcación: después de la rama auricular posterior, tras un trayecto de 4 a $5 \mathrm{~mm}$, el tronco principal se divide en cuatro ramas principales cuyo orden de aparición es: la del músculo digástrico, la cervical, la temporocigomática y la bucalmandibular. En los doce conejos todas las ramas se encontraron laterales a la vena facial posterior, afluente de la yugular externa, excepto la rama para el músculo digástrico que viaja en posición medial. En relación con la arteria carótida externa, todas las ramas del facial se hallaron laterales y separadas de ella por una delgada fascia.

La glándula parótida se halla sobre el ángulo de la mandíbula expandiéndose vía dorsal para cubrir el aspecto lateral de la base del oído externo y ventral por debajo de la mandíbula. Su pedículo vascular se localizó en el polo superior, y aunque estaba en estrecha relación con las ramas del nervio facial, éstas se ubican medial a su sustancia y no entre ella, permitiendo movilizar la glándula hacia la parte superior y posterior para exponer el nervio facial sin perjuicio de sus ramas ni de la glándula en sí misma (Figuras 2 y 3).

Ramas periféricas, la del músculo digástrico: es la primera de la bifurcación, pequeña, la única medial a la vena yugular externa que inerva el músculo digástrico, el cual está constituido sólo por un tendón redondo que se origina en el ligamento estilohioideo.

Rama cervical: es la más posterior e inferior, lateral a la vena facial posterior. Se dirige hacia el cuello por detrás del ángulo de la mandíbula y por delante de la porción mastoidea del músculo cleidomastoideo, para inervar los músculos platisma y estilohioideo.

Rama temporocigomática: en diez de los doce conejos, en ambos lados, tuvo un origen común, con longitud promedio de $3 \mathrm{~mm}$ ( 2 a $4 \mathrm{~mm}$ ) de la cual emergían dos divisiones: la temporal que se dirige hacia esta región en compañía de la arteria y la vena temporales superficiales y la cigomática, que va hacia

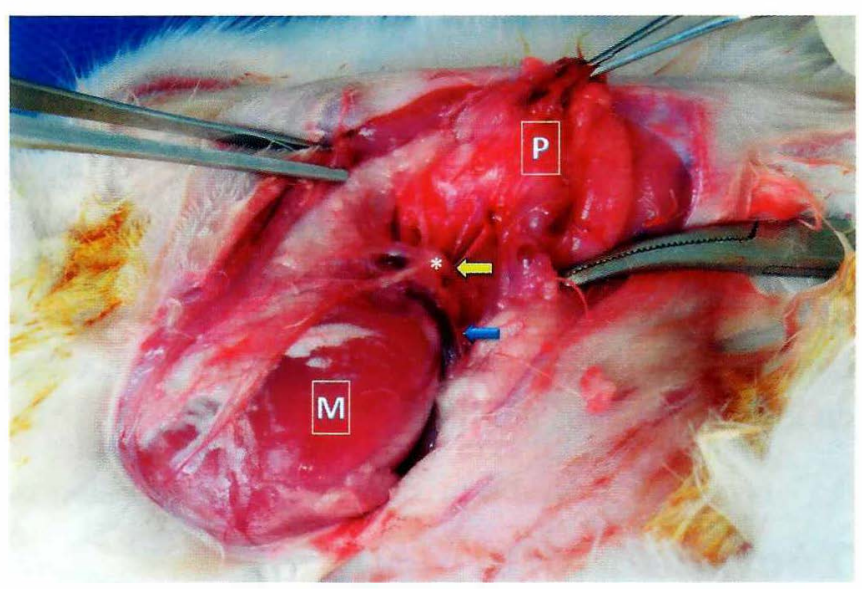

Figura 2. Nervio facial izquierdo. Se ha disecado en colgajo inferior: la piel, el tejido celular subcutáneo, el SMAS y la fascia superficial. Nótese el tronco principal (flecha amarilla) lateral a la vena facial posterior (flecha azul) con su bifurcación $(*)$ y la glándula parótida $(P)$ rechazada hacia las partes posterior y superior, con las ramas del facial sobre la cara lateral del músculo masetero (M).

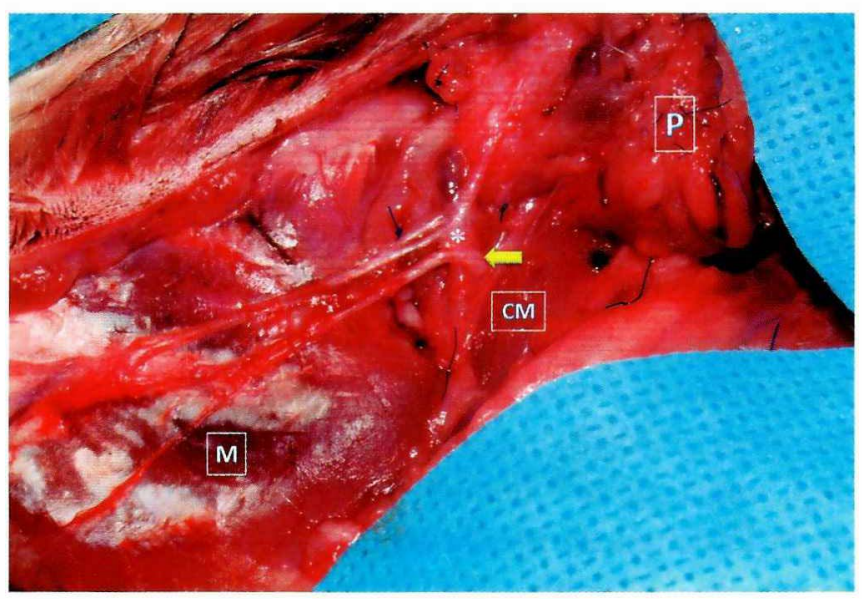

Figura 3. Nervio facial izquierdo. Se han ligado la vena facial posterior y sus afluentes. Se evidencia el tronco principal del nervio facial (flecha amarilla), con la bifurcación $\left(^{*}\right)$ y sus ramas, sin lesiones. P: glándula parótida; $M$ : músculo masetero; CM: músculo cleidomastoideo. 
la región orbitofrontal, lateral al canto externo del ojo. En dos conejos, cada división se originó del tronco principal. Estas son de difícil disección pues se encuentran mediales a las venas afluentes de la vena yugular externa y en cercanía al ojo, por tanto, no son susceptibles de manipulación experimental.

Rama bucalmarginal: la rama bucal tuvo un componente superior y otro inferior. En ambos lados se encontró en algunos un origen común y en otros partían del tronco principal por separado. El primero viaja sobre el músculo masetero y se dirige hacia la nariz y el labio superior. En su trayecto de 15 a $20 \mathrm{~mm}$ tiene múltiples anastomosis con el inferior. En todos los conejos se acompañó de una rama de V2 y su disección fue posible usando el microscopio quirúrgico. Medial a la rama bucal superior pasa el conducto de la glándula parótida, que perfora el músculo masetero para dirigirse a la cavidad oral. Esta rama es ideal para manipulación experimental por su fácil acceso, su tamaño y la ausencia de relación estrecha con vasos sanguíneos grandes. La rama bucal inferior también fue fácil de identificar cruzando la porción central e inferior del músculo masetero en un trayecto de $10 \mathrm{a}$ $15 \mathrm{~mm}$. Se bifurca como superior que se anastomosa con la bucal superior e inferior que se convierte en la rama marginal o mandibular y se dirige hacia el borde de la mandíbula. Tanto las ramas de la bucal superior como inferior o marginal, pasan laterales a la vena facial anterior antes de alcanzar la nariz, la boca o el mentón (Figura 4).

\section{Anatomía macroscópica del nervio auricular mayor}

Rama de C2 del plexo cervical superficial, contornea el borde posterior del músculo cleidomastoideo y asciende casi vertical hacia el pabellón auricular por detrás de la vena auricular posterior. Por la misma incisión descrita para el nervio facial, en el ángulo de la mandíbula y hacia la región cervical en relación con la glándula parótida, se encuentra un cojinete graso por debajo del cual se halla el nervio auricular mayor con su "vena centinela": la auricular posterior (Figura 5).
Se ha denominado así porque al seguir su trayecto hacia atrás, desde la entrada a la vena facial posterior por debajo del tronco principal del facial y rechazando el cojinete graso y la glándula parótida hacia adelante, se encontrará el nervio auricular mayor entre la cara posterior del músculo esternomastoideo y la cara anterior del músculo esplenio del cuello (Figura 6). Cerca del ángulo de la mandíbula, después de un segmento de 20 a $25 \mathrm{~mm}$ de longitud, se divide en dos, una anterior

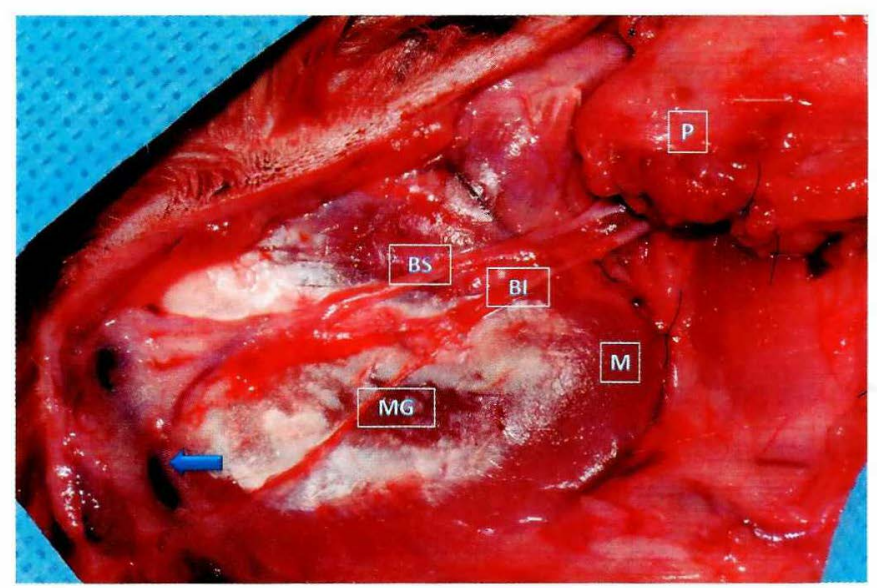

Figura 4. Ramas bucal superior (BS) e inferior (BI) con sus anastomosis. La marginal (MG) procede de la bucal inferior. Se observa la posición lateral respecto a la vena facial anterior (flecha azul), afluente de la vena yugular externa.

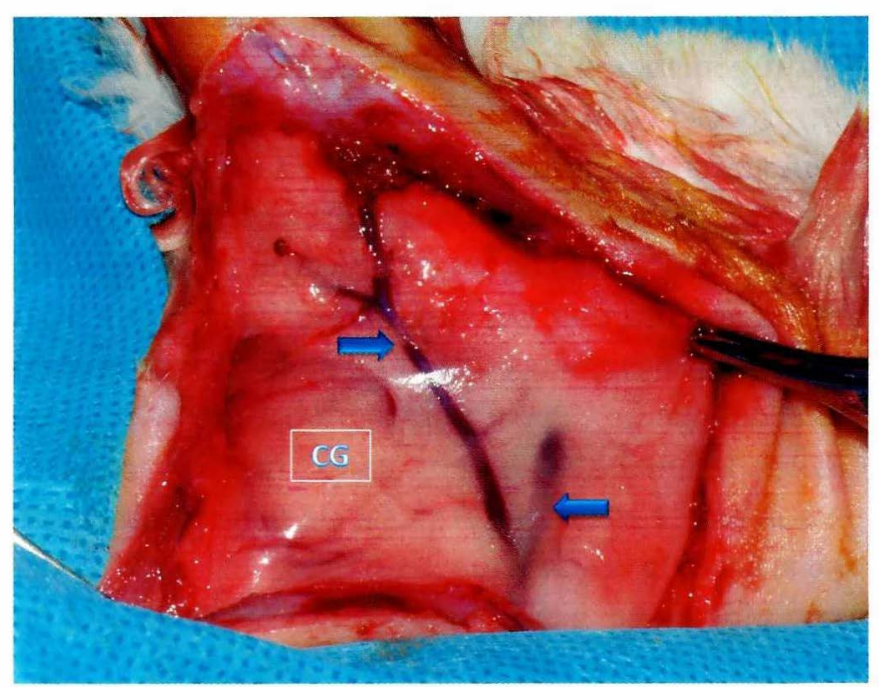

Figura 5. Cojinete graso (CG) subauricular, donde se encuentra la "vena centinela" (flecha azul a la derecha) del nervio auricular mayor, llegando a la vena facial posterior (flecha azul a la izquierda). 


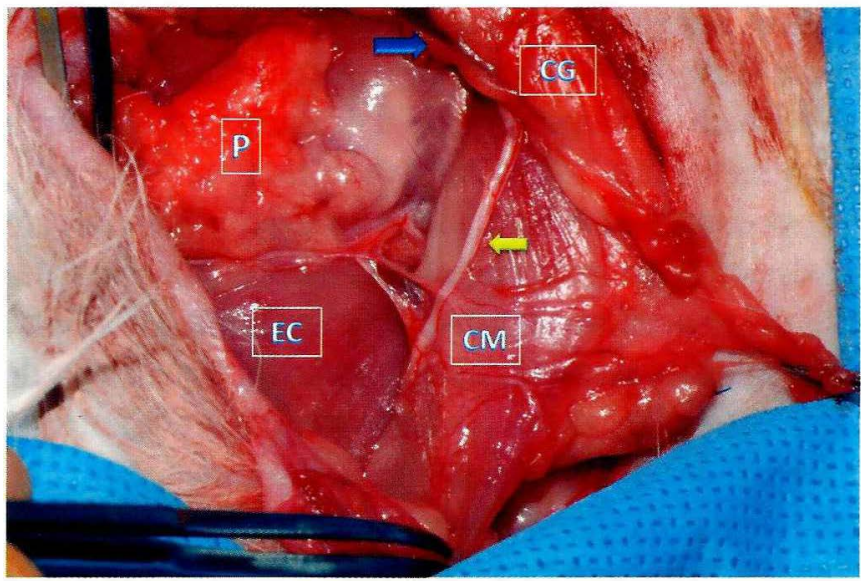

Figura 6. Nervio auricular mayor izquierdo (flecha amarilla), entre el músculo cleidomastoideo (CM) y el esplenio del cuello (EC). La parótida $(P)$ se ha rechazado hacia la región anterior $y$ el cojinete graso (CG) con la vena centinela o auricular posterior (flecha azul) hacia la parte posterior.

y otra posterior. La primera o aurículoparotídea se distribuye por la piel de la cara externa del pabellón y de la región parotídea. La posterior o aurículomastoidea se ramifica por la piel de la cara interna del pabellón y de la región mastoidea.

\section{Discusión}

Aunque la rata es de los animales más usados en experimentación, el conejo fue escogido como modelo para regeneración nerviosa, pues los estudios sobre este proceso en el nervio facial de la rata no semejan la situación clínica humana, debido a que el nervio es monofascicular y los axones en regeneración tienen menos probabilidad de tomar una dirección adecuada de crecimiento una vez que han entrado en un solo fascículo. ${ }^{1}$ En cambio, en cortes histológicos del nervio facial del conejo es posible comprobar que es polifascícular, al igual que en el humano ${ }^{3}$, que lo hace ideal para nuestros estudios. A pesar de no ser la meta de este artículo la descripción histológica de las ramas del nervio facial, se está trabajando en este propósito en otra investigación que se encuentra en curso.

De otro lado, el facial del conejo tiene relaciones anatómicas que fueron muy constantes en esta inves- tigación. Tanto el tronco principal como sus ramas son de fácil acceso quirúrgico, lo cual es ideal para su manipulación experimental. El principal se puede abordar siguiendo en forma retrógrada sus ramas periféricas o desde el ángulo de la mandíbula rechazando la glándula parótida, teniendo como reparo la vena facial posterior que se encuentra medial a él.

Es interesante notar que el nervio facial del conejo comparte con el humano algunos reparos de superficie como son el conducto auditivo externo y el ángulo de la mandíbula ${ }^{4}$; sin embargo, a diferencia del humano la porción extratemporal se encuentra por debajo de la glándula parótida y no entre ella, y el pedículo vascular de la glándula está en el polo posterosuperior, lo que permite separarla con facilidad del nervio sin lesionarla.

Este hallazgo fue descrito previamente por Bowden y colaboradores, quienes reportaron que existe una relación estrecha entre las ramas del facial, en especial la bucal superior, y el nervio trigémino, predominantemente con V2. Esta situación también la encontramos y debe tenerse en cuenta cuando se quieren evaluar los resultados funcionales después de la lesión del nervio facial. ${ }^{5}$

Por otro lado, esta es la primera descripción en la literatura respecto a la distribución anatómica y quirúrgica del nervio auricular mayor en el conejo, pues se encontraron las de otros nervios como el hipogloso y el vago. ${ }^{6,7}$

En el conejo es un nervio sensitivo con distribución polifascícular en número semejante al facial, disponible en el mismo campo quirúrgico y con reparos anatómicos constantes, características que lo hacen un modelo animal en potencia ideal para los estudios de regeneración nerviosa en la reconstrucción quirúrgica con injerto del nervio facial lesionado. Aunque los resultados en cuanto a patrones de regeneración nerviosa con el uso de nervios sensitivos como injerto para reconstruir nervios motores han sido controvertidos siguen siendo usados en virtud del escaso o nulo deterioro funcional tras su lesión. ${ }^{8}$ 
En conclusión, los modelos animales del nervio facial son necesarios para el desarrollo de nuevas técnicas de reparo quirúrgico. El del conejo es excelente para la regeneración nerviosa dada su configuración polifascícular semejante a la del humano y su fácil exposición quirúrgica. El auricular mayor es un nervio sensitivo útil como injerto, tanto por el mínimo efecto adverso tras su lesión, como por su accesibilidad en el mismo campo operatorio.

\section{Referencias}

1. Mattox DE, Felix H. Surgical anatomy of the rat facial nerve. Am J Otol. 1987;8(1):43-47.

2. Ellis JC, McCaffrey TV. Animal model for peripheral nerve grafting. Otolaryngol Head Neck Surg. 1984;92(5):546-50.

3. Captier G, Canovas F, Bonnel F, Seignarbieux F. Organization and microscopic anatomy of the adult human facial nerve: anatomical and histological basis for surgery. Plast Reconstr Surg. 2005;115(6):1457-65.

4. Malone B, Maisel RH. Chapter 2. Anatomy of the facial nerve. Am J Otol 1988;9(6):494-504.

5. Bowden RE, Mahran ZY. Experimental and histological studies of the extrapetrous portion of the facial nerve and its communications with the trigeminal nerve in the rabbit. J Anat. 1960;94:375-386.

6. Boyd JD. The sensory component of the hypoglossal nerve in the rabbit. J Anat. 1941;75(Pt 3):330-345

7. Evans DH, Murray JG. Histological and functional studies on the fibre composition of the vagus nerve of the rabbit. J Anat. 1954;88(3):320-337.

8. Lloyd BM, Luginbuhl RD, Brenner MJ et al. Use of motor nerve material in peripheral nerve repair with conduits. Microsurgery.2007;27(2):138-145. 